\title{
Educación e inserción laboral juvenil en Argentina ante la pandemia de Covid- 19
}

Education and youth labor insertion in Argentina in the face of the Covid-19 pandemic

Nery Facundo Rauch ${ }^{1}$

\section{Resumen}

El presente artículo se enmarca en la problematización sobre el vínculo entre juventud, educación y trabajo en el capitalismo contemporáneo, recurre al desarrollo del marco estructural del fenómeno en Argentina desde las décadas recientes a la luz de las repercusiones acaecidas con la irrupción de la pandemia de Covid-19.

A partir del eje central en la población juvenil, el artículo tiene por objetivo identificar las dimensiones del impacto de la pandemia de Covid-19 en la relación entre los jóvenes, la educación y su inserción en el mercado de trabajo. Se organiza en tres apartados: en primera instancia, indaga sobre las particularidades de la relación entre los jóvenes y el mercado de trabajo global y doméstico; posteriormente se aboca a profundizar sobre las implicancias prácticas que tiene la estructura social de la cual provienen los jóvenes en sus trayectorias educativas y laborales, las cuales contribuyen a reproducir inter-generacionalmente desigualdades; mientras que por último, se aborda la influencia del género y las particularidades en la sub-franja entre 18 y 24 años en la vinculación de los/as jóvenes con el trabajo.

Desde una metodología de diseño cuantitativo y tipo descriptivo, el artículo se nutre de aportes académicos provenientes de diversos campos disciplinares y de fuentes secundarias con indicadores estadísticos de organismos oficiales y privados sobre las características del empleo juvenil en los años recientes y ante el impacto de la pandemia por Covid-19. De esta forma, el artículo pretende contribuir en el análisis de una problemática que afecta crecientemente a nuestras sociedades reproduciendo estructuras de desigualdad intergeneracionales, desde el dimensionamiento de los desafíos que se presentan a partir del impacto del Covid-19 en la estructura sociolaboral juvenil de nuestro país.

Recibido: 26 de julio de 2021 Aceptado: 20 de diciembre de 2021 Publicado: 7 de enero de 2022

${ }^{1}$ Profesor en Ciencias Jurídicas, Políticas y Sociales y Licenciado en Ciencia Política (Universidad Nacional de Río Cuarto). Maestrando en Educación (Universidad Nacional de Quilmes). Becario Doctoral del Instituto de Investigaciones Sociales, Territoriales y Educativas (ISTE) - CONICET, Río Cuarto, Argentina. Correo electrónico: neryrauch@hotmail.com ID https://orcid.org/0000-0002-4226-5862 
Palabras claves: Inserción Laboral Juvenil, Educación, Trabajo Juvenil, Covid-19, Desigualdad.

\section{Abstract}

This article is part of the problematization of the link between youth, education and work in contemporary capitalism, it resorts to the development of the structural framework of the phenomenon in Argentina since recent decades in light of the repercussions that occurred with the irruption of the pandemic of Covid-19.

Starting from the central axis in the youth population, the article aims to identify the dimensions of the impact of the Covid-19 pandemic in the relationship between young people, education and their insertion in the job market. It is organized into three sections: in the first instance, it investigates the particularities of the relationship between young people and the global and domestic labor market; Subsequently, he delves into the practical implications of the social structure from which young people come in their educational and work careers, which contribute to the inter-generational reproduction of inequalities; while finally, the influence of gender and the particularities in the sub-group between 18 and 24 years old in the connection of young people with work is addressed.

From a quantitative and descriptive design methodology, the article draws on academic contributions from various disciplinary fields and secondary sources with statistical indicators from official and private organizations on the characteristics of youth employment in recent years and on the impact of the Covid-19 pandemic. In this way, the article aims to contribute to the analysis of a problem that increasingly affects our societies, reproducing structures of intergenerational inequality, from the dimensioning of the challenges that arise from the impact of Covid-19 on the youth socio-labor structure of our country.

Keywords: Youth Labor Insertion, Education, Youth Work, Covid-19, Inequiality.

\section{Introducción}

Se puede reconocer que, a pesar de la diversidad de los modos de producción acontecidos en la historia de la humanidad, el trabajo implicó un elemento indispensable tanto para el propio dinamismo histórico como para la realización personal de los individuos. A partir de la consolidación del capitalismo y su sistema productivo, el trabajo adquirió un carácter crecientemente objetivado- como los propios productos elaborados del mismo- y por ello, concebido como una mercancía sujeta a procesos de comercialización en la sociedad/ mercado. En este marco, se inscribe el nicho poblacional abocado a las actividades productivas de la sociedad, 
donde el trabajo "...es solo una de ellas, al lado de las actividades desarrolladas en las esferas doméstica, educativa, cultural, deportiva, sindical, política, etc" (Neffa, Panigo, Pérez y Giner, 2000, p. 10); y que comienza a los 15 años de edad ${ }^{2}$ (Ibid, p. 44).

En esta perspectiva, el objeto del presente trabajo se propone contribuir en el análisis de la relación entre los jóvenes, la educación y el mercado de trabajo, particularmente su inserción en éste. Con la pertinente aclaración que se entiende por juventud a la franja etaria conformada por personas entre los 15 y 29 años (Bonfiglio, Salvia, Tinoboras, y Van Raap, 2008), la cual además de ser la instancia donde generalmente se atraviesa la educación secundaria, conforma...

...una fase o período socialmente construido en el curso de la vida de las personas, que adquiere una entidad propia, durante el cual las sociedades ofrecen un conjunto de actividades y rituales que habilitan transiciones y pasajes, los cuales se van modificando a lo largo de las distintas épocas o etapas y brindan experiencias a los sujetos en su contexto generacional. (Cuervo y Wyn, 2014; Furlong, 2013; Bendit y Miranda, 2013, citado en Miranda, 2015, p. 82)

Donde se destaca que, entre dichas actividades, adquieren relevancia las relativas a la independencia económica y habitacional, sin constituirse en imperativos o modelos normativos para convertirse en personas adultas, fundamentalmente por la incidencia de la fragmentación territorial, desigualdades socioeconómicas o nuevas construcciones identitarias en la conformación de las transiciones juveniles. (Tilly, 2020; Saravi, 2004, 2014, citado en Miranda, 2015, pp. 93-94)

A fines metodológicos, corresponde advertir que el artículo recurre argumentalmente tanto a especialistas en el campo de estudio como al análisis de indicadores estadísticos de fuentes secundarias. En cuanto a su estructura, el artículo se organiza en tres apartados además de esta introducción y las reflexiones finales: en primera instancia se centra en la vinculación entre educación y trabajo en Argentina en tiempos del auge globalizador contemporáneo; posteriormente se profundiza en las implicancias prácticas que tiene la estructura social sobre las trayectorias educativas y laborales de los/as jóvenes; y por último, se aborda la incidencia del género y las particularidades de la franja que recién adquiere la mayoría etaria a la hora de insertarse en el mercado de trabajo.

\footnotetext{
2 No obstante, estas consideraciones conceptuales a nivel internacional, en las encuestas de hogares en Argentina, se hacen preguntas de mercado laboral a partir de los 10 años de edad.
} 


\section{Educación y trabajo juvenil en tiempos flexibles ${ }^{3}$}

A partir de las vicisitudes de la economía global posteriores a la década de 1970 y en paralelo al auge de la globalización de fines de siglo, el mundo del trabajo comenzaría a atravesar un proceso de cambios, tanto en los aspectos técnicos de sus modalidades, como en las características de la estructura productiva y las dinámicas del mercado laboral. Algo que se manifestaría fundamentalmente en una retracción de la demanda de puestos trabajo, lo que en el caso argentino se traduciría en un fuerte ascenso de los índices de desocupación (8,4\% en 1989 a 21,5 en 2002) y subocupación (9,3\% en 1989 a 18,6\% en 2002) durante la década del 90 y principios del 2000 (Rapoport, 2010, pp. 753 y 884).

Dichas dificultades para los sectores poblacionales económicamente activos se agravarían en la franja juvenil, al manifestar mayores dificultades e inestabilidad para incorporarse al mundo del trabajo, ya sea de forma parcial o completa. Según Miranda (2008), el desempleo joven alcanzaría su máximo histórico en Argentina en el año 2002 con un $36,9 \%$ (p. 187). Y si bien estos guarismos se redujeron a partir de la reactivación económica de nuestro país, con el fin de la convertibilidad y los elevados precios internacionales de los commodities ${ }^{4}$, el mercado laboral juvenil preservaría sus características estructurales de fuerte vulnerabilidad y crecimiento ocupacional endeble, sobre todo si se compara con el resto de la población económicamente activa (P.E.A).

El advenimiento de este modo productivo flexible del capitalismo contemporáneo nos presenta un escenario laboral donde -sobre todo- los jóvenes deben enfrentar especiales obstáculos para alcanzar una inserción laboral de calidad, ingresan al mercado de trabajo en condiciones de inestabilidad, sin protección social y con bajos ingresos (Organización Internacional del Trabajo, 2020, p. 2), al punto de que los jóvenes tienen tres veces más probabilidades de estar desempleados que los adultos (Ibid. p. 1). Lo que promueve ocasionalmente desaliento laboral y exclusión social cuando los/as jóvenes deben transitar períodos prolongados de desempleo y precariedad laboral (Bonfiglio, Salvia, Tinoboras, y Van Raap, 2008, p. 6).

\footnotetext{
${ }^{3}$ Se apela al termino en un sentido amplio, abarcando desde la propia reconversión de las dinámicas productivas orientadas por la constante adecuación a las fluctuaciones del mercado como a las de las relaciones laborales y/o sociales. Constituyendo a la flexibilidad como un dispositivo de control (o autocontrol) que erigiéndose bajo un imperativo de promoción de libertad conduce a los/as individuos a una constante asunción de autoevaluación. (Beck, 1998; Sennett, 2000; Dardot y Laval, 2013)

${ }^{4}$ Constituyen bienes o productos básicos, cuyo destino es un fin comercial, y entre sus características poseen escaso o ningún valor agregado, es materia prima sin procesar o sin una característica diferenciadora respecto a otros productos del mercado.
} 
Lo paradójico resulta que, en paralelo al incremento de las dificultades de los jóvenes para incorporarse al mundo del trabajo, se daría un creciente proceso de acrecentamiento de "inversión educativa” por parte de este grupo en nuestro país y en gran parte del mundo. Según el informe sobre "Tendencias mundiales sobre empleo Juvenil 2020" de la Organización Internacional de Trabajo la tasa de participación de los jóvenes en la fuerza de trabajo presenta una caída sostenida en las últimas décadas. Entre 1999 y 2019 la población mundial juvenil aumentó de 1000 millones a 1300 millones de personas, sin embargo, la fuerza de trabajo ha disminuido de 568 millones a 497 millones. Esto sucede fundamentalmente por la creciente matriculación en instituciones de educación secundaria y superior, lo que en muchos países se traduce en una fuerza de trabajo más calificada (p. 2).

En lo que refiere a la experiencia doméstica, el proceso de inversión educativa se dio primera instancia por una "obligatoriedad subjetiva” (década de los `80) y luego por una obligatoriedad normativa, producto de las reformas educativas de los 90(Ley Federal de Educación, 1994) y del nuevo milenio (Ley de Educación Nacional, 2006) (Miranda, 2008, p. 188).

Este fenómeno es el que ha contribuido de forma gravitante a que en la actualidad no se considere socialmente aceptable que los jóvenes abandonen la educación antes de alcanzar la mayoría de edad. En efecto, “...las menores oportunidades laborales y los nuevos requerimientos ocupacionales han significado que en la actualidad se considere que el mejor lugar para los menores de 18 años es la escuela secundaria” (Miranda, 2008, p. 186). En este sentido, se manifiestan quienes abogan que el proceso de transición de los jóvenes entre la escuela media y el mundo del trabajo sería más exitoso si se establecen “...transformaciones innovadoras en materia educativa y políticas activas de intermediación, capacitación y promoción del empleo juvenil (sistemas de pasantías, flexibilización laboral, etc.), se acierte en reconstruir de manera virtuosa el vínculo entre la escuela y el mercado laboral" (Bonfiglio, Salvia, Tinoboras y Van Raap, 2008, p. 7), ya que "en casi todos los ámbitos se ha asumido que el principal escollo que enfrenta la demanda laboral de los jóvenes es el déficit en "capital humano"... asociándose casi exclusivamente su alcance y nivelación a los problemas de educación” (Ibid, p. 8). Sin embargo, un análisis que se acote a enfocar el problema de la empleabilidad de los jóvenes a un problema estrictamente educativo, relega $\mathrm{u}$ omite las características estructurales de desigualdad entre los jóvenes que forman parte de la composición educativa nacional -regional y global-, originada tanto por la inestable y endeble estructura económica de nuestro país como las propias transformaciones en el mercado de trabajo globalizado (Weller, 2007, p. 63). 
Resulta oportuno describir someramente las principales dificultades que reconoce Jürgen Weller (2007) para la inserción laboral juvenil. En primera instancia, él observa una aceleración del cambio en los mercados como consecuencia de la integración global y los avances tecnológicos, lo que afecta también al funcionamiento del mercado de trabajo, algo que facilitado por reformas legales, contribuyó a un fuerte aumento de la inestabilidad laboral, sobre todo en los/as jóvenes. Otro elemento que complica la inserción laboral juvenil es la marcada, y creciente, segmentación socioeconómica, reflejo y origen de la elevada desigualdad en Argentina, y América Latina. En efecto, el trasfondo familiar determina en gran medida las perspectivas laborales de los jóvenes, pues influye en las oportunidades de acumular capital humano (acceso a educación y capacitación de buena calidad), capital social (relaciones sociales basadas en la confianza, la cooperación y la reciprocidad) y capital cultural (manejo de los códigos establecidos por la cultura dominante). (p.63)

Aclarado este aspecto, y en consonancia con Bonfiglio, Salvia, Tinoboras y Van Raap (2008) se debe remarcar que:

Los progresos o retrocesos en materia de inclusión educativa y laboral responden en primera instancia a las condiciones generales de desarrollo e inclusión que presenta el sistema económico y social de nuestro país, y no exclusivamente a las políticas compensadoras más o menos virtuosas que los gobiernos puedan emprender en procura de prolongar la escolaridad de los jóvenes, aumentar sus competencias o facilitar su entrada al mercado laboral. (p.9)

Y por ello, a continuación se abocará a reflexionar sobre cómo repercute en materia educativa y laboral el origen social de los/las jóvenes.

\section{Las particularidades de la inserción laboral joven y el factor del origen social}

Las dinámicas productivas y sociales del capitalismo contemporáneo han impactado sustancialmente en la población juvenil, destacándose en su tránsito hacia la adultez, en este marco, su incorporación al mercado de trabajo. En continuidad con los desarrollos de Weller, "Inserción laboral de jóvenes: expectativas, demanda laboral y trayectorias" (2006), se pueden observar diferentes dificultades que se presentan particularmente en este sector poblacional a la hora de incursionar en el mundo laboral, entre ellas se destacan: a) Si bien actualmente los jóvenes tienen niveles más altos de educación formal que las cohortes etarias anteriores, el problema radica en que los avances de la cobertura educacional son insuficientes para alcanzar la equidad 
y el desarrollo de la región y su calidad es inadecuada. Donde puede observarse que los sistemas de educación y formación para el trabajo han sido débiles en abordar los cambios productivos y socioculturales recientes, de manera que no facilitan la transición al mundo laboral (Ibid. p. 2); b) Los jóvenes otorgan una alta valoración al trabajo en sí, pero sus experiencias con empleos concretos suelen ser frustrantes, ya que son muchos los jóvenes que informan sobre condiciones que no estimulan a aprovechar el potencial que promete el trabajo para el desarrollo individual y social de los jóvenes (Ibid. p.3); c) Las mujeres jóvenes desarrollan en forma cada vez más marcada un desarrollo personal autónomo, y para independizarse de los roles tradicionales estrechamente vinculados con el hogar el empleo juega un papel clave. Sin embargo, se enfrentan a problemas especiales de inserción laboral, siendo este problema particularmente grave para mujeres con educación formal baja, las cuales tienen escasas oportunidades de empleo productivo (Ibid. p. 5); d) La creciente importancia de la combinación del trabajo con estudios puede generar tensiones negativas al afectar el rendimiento en ambos campos, o positivas al abrir el acceso a oportunidades de otra manera cerradas. En la medida en que el trabajo afecta el rendimiento escolar, debido a extensas o extenuantes jornadas laborales que restringen la capacidad de aprendizaje, este aumento representa una tendencia negativa que hipoteca el futuro de los jóvenes (Ibid. p.5); e) Los jóvenes viven la tensión entre un discurso meritocrático y una realidad del mercado de trabajo en que los contactos personales y las recomendaciones frecuentemente juegan un gran papel para el acceso a empleos atractivos (Ibid. p. 6); f) El mercado exige experiencia laboral pero para muchos jóvenes que buscan trabajo por primera vez es sumamente difícil acumular esta experiencia y en similar medida, el mercado no reconoce la experiencia generada en muchas de las ocupaciones accesibles para jóvenes de bajo nivel educativo, por lo que, para este grupo, es casi imposible generar una trayectoria laboral ascendente (Ibid. p.6).

En este sentido, en la trayectoria educativa de los jóvenes de menores ingresos, con frecuencia se plantea la necesidad del núcleo familiar de que comiencen a contribuir económicamente en el sostenimiento familiar, ya sea mediante trabajos temporales o en algunos casos, el definitivo abandono de su trayectoria educativa para abocarse a un trabajo de tiempo completo, el cual, por las propias características que se han desarrollado, en la mayoría de los casos son informales (Miranda, 2008, p. 197; Bonfiglio, Salvia, Tinoboras y Van Raap, 2008, p. 24).

Por otro lado, si bien se reconoce que "los jóvenes con credenciales secundarias tienen 2 veces más probabilidades de acceder a un empleo estable y protegido que sus pares sin credenciales o credenciales inferiores" (Bonfiglio, Salvia, Tinoboras y Van Raap, 2008, p. 22), hay que destacar que las diferencias en base al origen social de los 
adolescentes no solo se presentan en relaciona a la culminación o no de su trayectoria educativa, sino que también influye sobre las propias credenciales que emite la institución a la que concurrieron, ya que la creciente estructura fragmentada del sistema educativo termina conduce a que una misma titulación puede representar diferentes tipos de capital cultural incorporado, y genera de esta forma una valoración desigual a la hora de insertarse laboralmente entre personas que provienen de instituciones diferentes.

En materia laboral, también incide de forma directa el origen social de los jóvenes, no solo por las características particulares de las trayectorias educativas que se han analizado en los párrafos anteriores, sino porque dichas desigualdades también influyen en los años posteriores a la culminación de la educación secundaria. A la hora de afrontar su vida luego de concluir la educación obligatoria, los jóvenes de sectores medios y altos, en su mayoría, deciden continuar sus estudios superiores, mientras que los de los sectores de ingresos bajos, deciden intentar insertarse inmediatamente en el mercado de trabajo, (Miranda, Corica, Aranciba y Merbilhaá, 2014, pp. 135136). En segunda medida, los jóvenes que deciden incorporarse al mercado laboral, también presentan situaciones desiguales en base a su origen social, ya que los jóvenes de sectores bajos tienen más dificultades para incorporarse al mundo del trabajo que los de sectores medios o altos, por insuficiencia de Capital Cultural y sobre todo, de Capital Social (Fridman y Otero, 2015, p. 176); mientras que por otro lado, se presenta otro de los grandes flagelos que atraviesa la juventud contemporánea, que es el de la informalidad o precariedad del empleo, el cual manifiesta también un mayor impacto en los sectores de menores recursos (Bonfiglio, Salvia, Tinoboras y Van Raap, 2008, p. 23). 
Gráfico 1. Tasas de actividad, empleo y desempleo juvenil (15-29 años) por nivel de ingresos familiares ${ }^{5}$. Argentina, 2016-2020. ${ }^{6}$

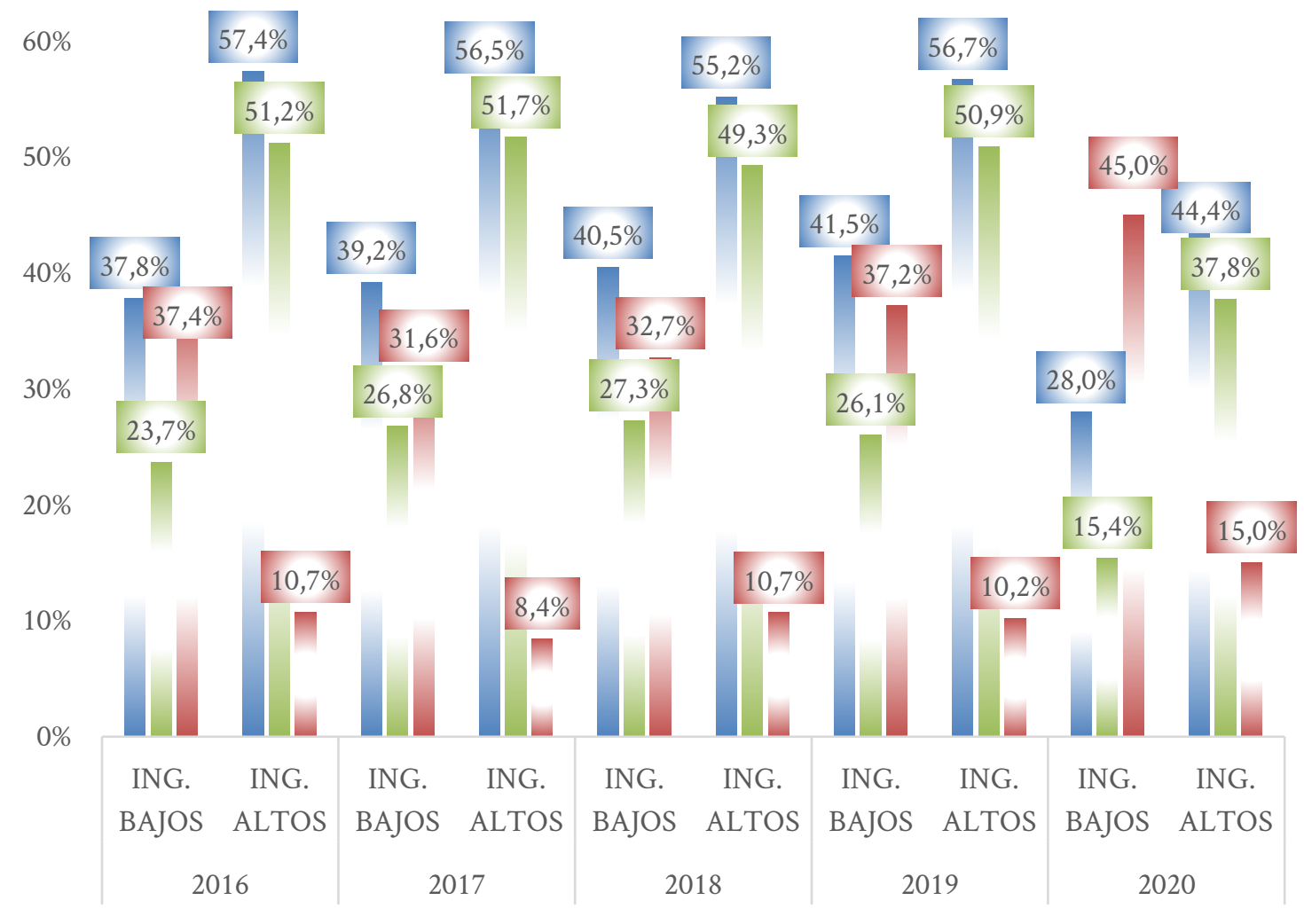

$\square$ TASA DE ACTIVIDAD $\square$ TASA DE EMPLEO $\square$ TASA DE DESEMPLEO

Fuente: Elaboración propia en base a la EPH7 - INDEC.

De esta forma, se puede reconocer un amplio abanico de dificultades que afrontan los/as jóvenes para su inserción en el mundo del trabajo, y a partir de ello, resulta oportuno profundizar el análisis sobre cuál es su relación con el origen social de los/as jóvenes. Donde se destaca que producto del impacto de la pandemia por Covid-19, el 34\% de los/as jóvenes de entre 18 y 30 años provenientes de los tres deciles más pobres de nuestro país perdió su empleo, mientras que en los tres deciles de mayores ingresos de la misma fracción poblacional la perdida de trabajo solo implicó al 3\% (Sosa, Smith y Romano, 2021, p. 8).

Este dato estadístico, expone el impacto de la pandemia de Covid-19 en materia laboral de la juventud en base a la incidencia del sector social de origen, a fin de dimensionar que, si bien como grupo poblacional la juventud se ha conformado como

\footnotetext{
${ }^{5}$ Ingreso total familiar del total de la Encuesta Permanente de Hogares. Se considera con Ingresos Bajos a los deciles uno y dos, e ingresos altos a los deciles nueve y diez.

${ }^{6}$ Todos los registros anuales son del relevamiento correspondiente al 2 do Trimestre.

${ }^{7}$ Encuesta Permanente de Hogares.
} 
un sector vulnerable en materia de empleo, hay que distinguir que el grado de afectación en esto, varía en relación a las condiciones sociales del hogar de procedencia.

\section{La incidencia del género y de los primeros años de la juventud}

Tal como se advierte, el origen social de los/as jóvenes conforma un importante condicionante para su desempeño educativo y laboral presente y futuro, pero los factores de incidencia no acaban allí. Ya que como desarrolla Jürgen Weller (2006) abordado en subtítulos precedentes- las dificultades que se le presentan a la juventud al momento de incorporarse al mundo laboral son diversas, destacando entre ellas el género y la experiencia o vínculos sociales que disponen para ingresar a un trabajo.

La incidencia del género de los/as jóvenes es relevante para obtener empleo, afectando particularmente a las mujeres según observan especialistas en el campo. Scarano, Ascencio, Sacco, Gabilondo, Strada y Ruiz Elvira (2019) destacan que "Las mujeres y los varones tienen perfiles de inserción diferentes en el mercado de trabajo: las mujeres, tienen una menor tasa de actividad y trabajan menos horas en promedio que los varones, lo que tiene un efecto directo sobre los ingresos laborales” (Ibid. p.3), esto está directamente relacionado a lo que se reconoce como trabajo reproductivo, el cual es mayoritariamente desempeñado por las mujeres, no solo en materia de actividades de orden doméstico sino en tareas de cuidados de menores y de personas mayores (Ibid. p.8). En este sentido, según informes de la Dirección Nacional de Economía, Igualdad y Género (2020) el 76\% de dichas actividades recaen sobre ellas; mientras que solo el 24\% en los hombres (Sosa, Smith y Romano, 2021, p. 7).

Además de esto, existen otros factores que repercuten negativamente en la inserción o desempeño laboral femenino, destacándose mayores tasas de informalidad laboral, lo que implica menores ingresos y mayor vulnerabilidad en materia de derechos laborales y de acceso a cobertura jubilatoria. En similar sentido se presenta el perfil de inserción laboral diferencial entre hombres y mujeres, ya que éstas se insertan de forma más intensiva en actividades denominadas "profesiones del cuidado" (docencia, salud, y trabajo doméstico), "reproduciendo en el mercado laboral la división sexual del trabajo que opera al interior de los hogares. Mientras que sólo $6 \%$ de los varones se dedica a estas actividades, entre las mujeres asciende a $32 \%$ (CEPA, 2018 en Scarano, Ascencio, Sacco, Gabilondo, Strada y Ruiz Elvira, 2019, p. 3). Por último, se presenta la dificultad de las mujeres en lo que se reconoce como "techos y paredes de cristal" y "pisos pegajosos", donde "las mujeres se encuentran sobrerrepresentadas en los niveles inferiores de las organizaciones y subrepresentadas en los trabajos de mayor responsabilidad” (Ibid, p. 3). 
Tal como se advierte, el impacto de la desigualdad entre mujeres y hombres (el caso de la población LGBTI+ amerita un trabajo en particular, desde el reconocimiento de la frecuente omisión en los análisis cuantitativos de organismos oficiales) es gravitante en el conjunto de la población, según el Instituto Nacional de Estadística y Censos (INDEC), en el primer trimestre de 2021 el desempleo total alcanzaba al 10,2\% de la población, mientras que en particular lo hacía en un 8,5\% en los hombres y ascendía al 12,3\% en las mujeres (p. 8). Donde expone mayor agudeza en el caso de la población juvenil, donde la desocupación en mujeres de entre 15 y 29 años registró de $24,9 \%$, y un $17 \%$ entre los hombres, observándose así, una diferencia de casi $8 \%$ entre personas de diferentes géneros e idéntica franja poblacional. Y si bien estas tasas resultan evidentes, corresponde advertir que presentan una mejoría con respecto a los del segundo trimestre de 2020 -durante el auge de las restricciones por la pandemia de Covid-19-, cuando la pérdida de puestos de trabajo en idéntica franja poblacional alcanzó al 28,5\% de las jóvenes, mientras que en el caso de los varones fue del $22,7 \%$.

Gráfico 2. Tasas de desempleo juvenil (15-29 años) por género. Argentina, 2016$2020^{8}$

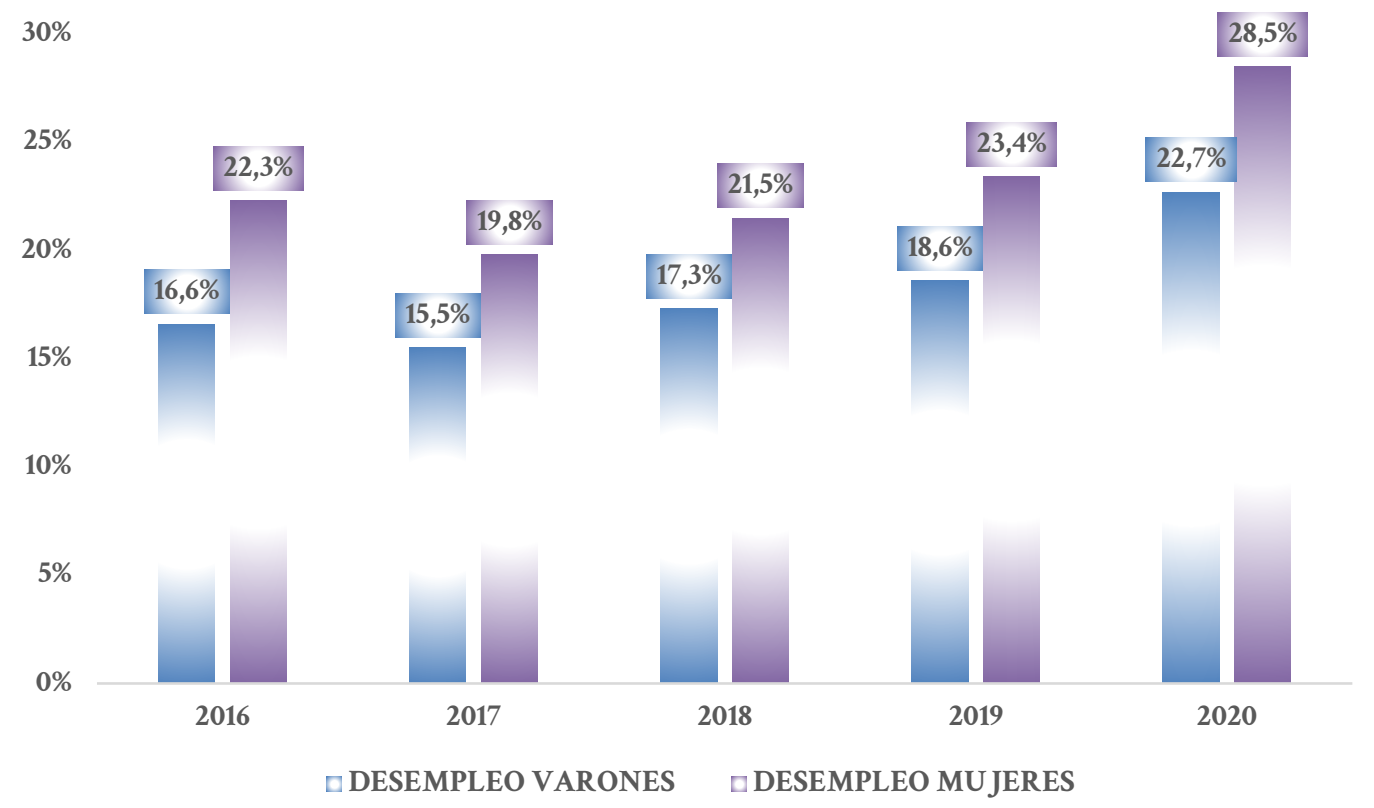

Fuente: Elaboración propia en base a la EPH- INDEC.

Otro aspecto que marca incidencia en los procesos de incorporación al mercado de trabajo de los/as jóvenes, radican en que instancia de la juventud se encuentran, ya que quienes padecen mayores complejidades es la sub-franja de los 18 a 24 años;

\footnotetext{
${ }^{8}$ Todos los registros anuales son del relevamiento correspondiente al 2 do Trimestre. 
fundamentalmente porque con frecuencia aún no han culminado su escolaridad obligatoria o lo han hecho recientemente, cuentan con escasa/nula experiencia laboral o carecen de capital social que facilite su ingreso al ámbito laboral, lo que radicaliza las tensiones planteadas por Jürgen Weller (2006, p.6).

Gráfico 3. Evolución de la tasa de desempleo por franjas etarias. Argentina, 2016$2020^{9}$.

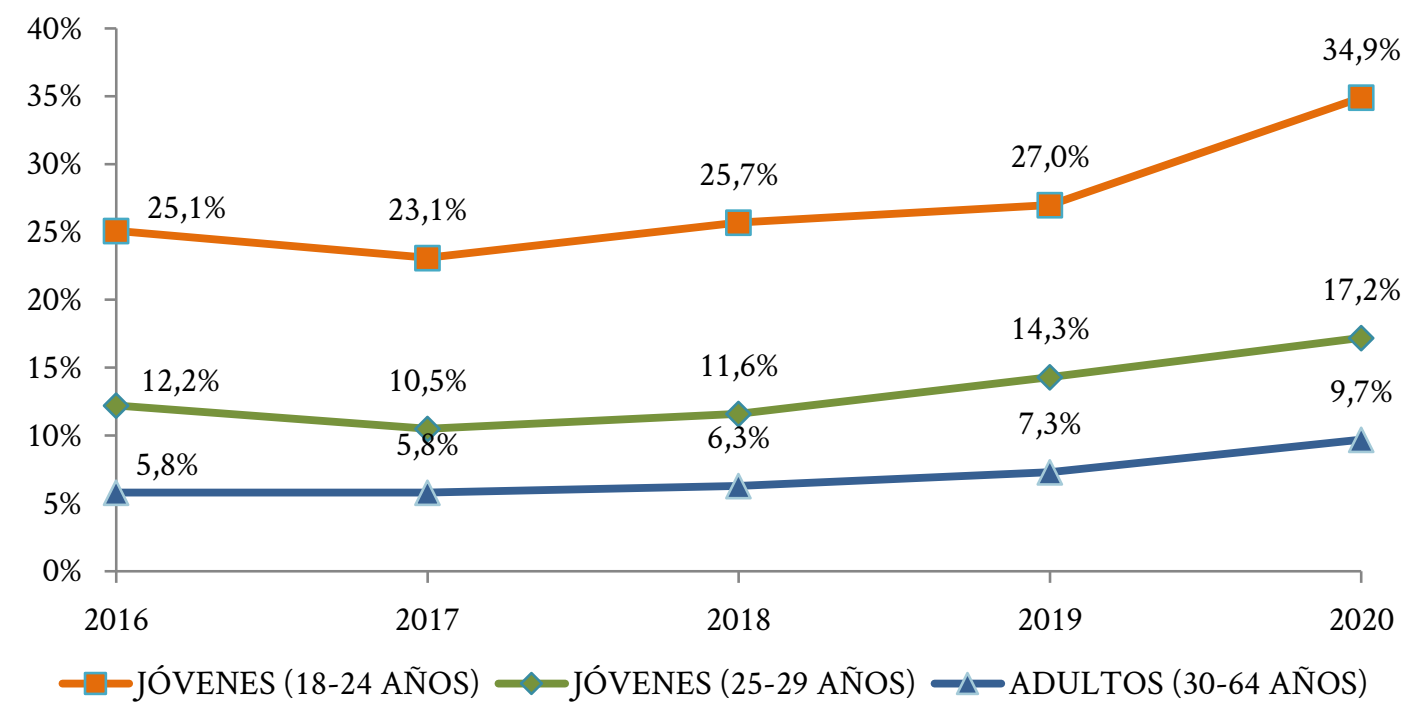

Fuente: Elaboración propia en base a la EPH- INDEC.

A partir del gráfico precedente, se puede observar que la vinculación al mercado de trabajo presenta notables condicionantes en relación a la franja etaria de las personas. En este sentido, se observa que las posibilidades de ser desempleado/a es casi cuatro veces superior si se encuentra en la franja entre 18 y 24 años con respecto a quienes tienen más de 30 años y del doble con respecto a quienes se encuentran en la franja entre 25-29 años. Si bien en el período analizado los índices no exponen un agravamiento de la situación para la franja 18-24 años en términos comparativos con los restantes grupos poblacionales aun ante el impacto de la pandemia de Covid-19, fundamentalmente por el empeoramiento en el nivel de desempleo de las franjas 2529 años y 30-64 años. No obstante, si se observa un notable incremento en las brechas reales entre los grupos en su incidencia porcentual con la llegada del Covid-19; en 2019, el desempleo afectaba en 19,7\% más a los/as jóvenes de entre 18-24 años que a la población entre 30 y 64 años, y en 12,7\% más que al grupo entre 25 y 29 años; mientras que, en el 2020, la diferencia alcanzó el 25,2\% con respecto a los/as adultos/as y el 17,7\% con el grupo de entre 25-29 años. En relación a ello, Sosa, Smith y Romano (2021) advierten:

\footnotetext{
${ }^{9}$ Todos los registros anuales son del relevamiento correspondiente al 2 do Trimestre. 
El subgrupo de jóvenes de entre 18 y 24 años se vio notablemente más afectado, perdiendo el $40 \%$ de los puestos de trabajo durante el momento más crítico de la pandemia ( $2^{\circ}$ trimestre). En tanto en el tramo de entre 25-30 años la caída fue de 20\%. (p.7)

Y aunque la situación mejoró en el transcurso de los meses posteriores, y se observa una recuperación del empleo en este tramo etario -al igual que en los demás, a fines de 2020 aún persistían un 30\% menos de ocupados de 18-24 años que antes del inicio de la pandemia (Ibid. p.7). Lo que deja en evidencia, que los primeros años posteriores a la edad teórica (Terigi, 2007, p.12) de culminación de la escolaridad obligatoria resultan notablemente adversos para incorporarse al mundo laboral para los/as jóvenes.

\section{Reflexiones finales}

El auge de la globalización contemporánea en el orden económico y social internacional condujo a una creciente flexibilización de las relaciones laborales en la estructura productiva. Lo que aparejó tanto mayores índices de desigualdad en nuestras sociedades como crecientes dificultades para los sectores laborales, amparadas por reformas legales y sustentadas comunicacionalmente, posibilitaron contratos temporales, facilidades en despidos y reducciones salariales en virtud de la "competitividad internacional".

Los crecientes índices de desempleo y trabajo informal producto de las flexibilizaciones laborales afectaron negativamente en el conjunto de la población, pero con mayor impacto en los sectores históricamente vulnerabilizados, entre ellos la juventud, que ha visto dificultársele crecientemente sus posibilidades de insertarse y desenvolverse en el mundo laboral; en simultaneidad con la exigencia de incrementar los niveles obligatorios de escolarización, algo que al relegar la modificación de las condiciones sociales de los/as jóvenes, propugnó una estructura educativa con creciente fragmentación interna y que reprodujo en el plano educativo las desigualdades que caracterizan a la estructura social Argentina. (Tiramonti, 2005; Tuñon y Halperin, 2010)

En este sentido, se puede observar que tanto los aspectos educativos como laborales encuentran un condicionamiento en la procedencia social de la juventud, no solo en los casos de deserción educativa o ingreso temprano al mundo del trabajo (llevándolo de forma paralela a su periodo de escolarización), sino inclusive una vez culminados los estudios secundarios, producto de la fragmentación educativa y de sus credenciales, o del capital social que posee la familia de origen, los cuales producen 
que a los sectores de menores recursos se les dificulte encontrar trabajo o que este sea de buena calidad con respecto a los sectores de medianos y altos ingresos. Lo que, desde el advenimiento de la pandemia si bien resultó un hecho extraordinario, expuso las desigualdades entre jóvenes de diversos origines socioeconómicos, tanto en la voluntad de participar en el mercado de trabajo, como de obtener empleo o resultar desempleado. Lo que contribuye en la conformación de un estrecho vínculo entre desigualdad social, educativa y laboral; con sus potenciales implicancias de reproducir un círculo virtuoso para los de sectores más altos ingresos, y vicioso para los/as de procedencia más baja en la pirámide socioeconómica. (Tenti Fanfani, 1995; Tiramonti, 2007)

Otro aspecto de relevancia a la hora de insertarse o desenvolverse en el ámbito laboral lo conforma el género de los/as jóvenes, donde para las mujeres resulta más complejo producto de diversas estructuras patriarcales de la sociedad, como sucede con la división de tareas domésticas o de cuidados, que no solo implican menor tiempo disponible para el trabajo fuera del hogar, sino que también prefiguran las características de la potencial inserción, ya que ellas lo hacen de forma más intensiva en actividades del cuidado como docencia, salud, y trabajo doméstico. Algo que con la llegada de la pandemia de Covid-19 no solo incrementó los indicadores de desempleo entre la juventud de ambos géneros (en consonancia con los indicadores generales), sino que amplió la brecha entre varones y mujeres con respecto a las desigualdades de los años previos.

Los primeros años luego de superar la mayoría de edad (18 años) también inciden en las dificultades para incorporarse al mundo laboral, muchas veces aparejados a una insuficiente formación académica (requisito incuestionable en las nuevas dinámicas del mercado laboral), carencia de experiencia laboral, malas experiencias laborales previas, o simplemente por carecer vínculos sociales para ocupar un puesto de trabajo vacante. En similar medida que en el factor previo, la subfranja entre los 18 y 24 años presenta índices más elevados de desempleo, subempleo y precariedad laboral que la de entre 25 y 29 años y más aún que entre la población que supera los 30 años; mientras que si bien las desigualdades porcentuales entre franjas etarias en materia de desempleo se acotaron levemente con la llegada de la pandemia en 2020, esto no resulta por una mejoría en la situación laboral de la fracción juvenil inferior, sino por el empeoramiento de la situación en los restantes grupos poblacionales.

Para concluir, corresponde reiterar que este articulo ha pretendido contribuir en el abordaje de una problemática con nutrido desarrollo académico en el campo de la sociología educativa argentina, se advierte que los factores que inciden en la empleabilidad juvenil no han sido abordados en su totalidad ni con la profundidad 
que ameritan, resultan pendientes -entre otros- los aspectos ligados a las trayectorias educativas (Corica y Otero, 2018; Garino, 2020), las instituciones y credenciales educativas (Jacinto, 2018), o donde se desempeñan los/as jóvenes o las características del sector de la demanda en el mercado laboral(Fridman y Otero, 2019; Maynard, 2019), lo cual implicará desarrollos analíticos futuros. Se remarca, en total coincidencia con Bonfiglio, Salvia, Tinoboras y Van Raap (2008), que si bien la educación es una variable clave para mejorar las perspectivas laborales de los/as jóvenes, esta no conforma ni la primera ni la última causa de los problemas de empleo, sino que es más bien “...una consecuencia o un eslabón en un círculo de reproducción y de transmisión intergeneracional de situaciones de pobreza y de precariedad cada vez más difíciles de modificar" (p.25), que se ha visto agudizada por la emergencia sanitaria de la pandemia de Covid-19 y sus implicaciones económicas. Lo que amerita maximizar el trabajo por políticas públicas que den respuesta a esta problemática, hacia una revisión integral de la vinculación entre el sistema educativo y el mundo del trabajo, fundamentalmente a partir de desandar la estructura legal y económica que condujo la situación plasmada en el artículo.

\section{Referencias}

Beck, U. (1998). La sociedad del riesgo. Hacia una nueva modernidad. Barcelona: Paidós. Bonfiglio, J., Salvia, A., Tinoboras, C., y Van Raap, V. (2008). "Educación y trabajo: Un estudio sobre las oportunidades de inclusión de los jóvenes tras cuatro años de recuperación económica”. En Salvia, A. (Comp.) Jóvenes promesas. Buenos Aires: Editorial Miño y Dávila.

Centro de Economía Política Argentina. (2017). Jóvenes y empleo, en números. [Informe]. Recuperado el 11 de julio de 2021, de https://gallery.mailchimp.com/e9c6f62a4dc825f6a9dab4e88/files/2423ceeb25c1-4986-b526-7c515c8b0806/J\%C3\%B3venes_y_empleo.pdf

Centro de Estudios Metropolitanos. (2020). Desempleo Joven y Políticas De Empleo. Documentos CEM N25. Universidad Nacional Arturo Jauretche, Universidad Metropolitana de la Educación y el Trabajo y Universidad Nacional de Hurlingham. Recuperado el 16 de junio de 2021, de http://estudiosmetropolitanos.com.ar/wpcontent/uploads/2020/03/Desempleo-joven-y-pol\%C3\%ADticas-deempleo.pdf

Corica, A. y Otero, A. (2018). Transiciones juveniles: un análisis sobre el vínculo educación y trabajo de jóvenes egresados de la educación obligatoria argentina. 
Ultima década $N^{\circ}$ 26(48), 133-168. Recuperado el 13 de noviembre de 2021, de https://dx.doi.org/10.4067/S0718-22362018000100133

Laval, C. y Dardot, P. (2013). La nueva razón del mundo: Ensayo sobre la sociedad neoliberal. Barcelona: Gedisa.

Fridman, V. y Otero, A. (2015). "De estudiantes a trabajadores. Un análisis sobre trayectorias ocupacionales de jóvenes argentinos durante la última década” en Miranda (Cord.) Sociología de la educación y transición al mundo del trabajo: juventud, justicia y protección social en la Argentina contemporánea. Ciudad Autónoma de Buenos Aires: Teseo

----. (2019). Cooperativismo e inclusión laboral joven. Una experiencia argentina actual. Santiago del Estero: Universidad Nacional de Santiago del Estero. Facultad de Humanidades y Ciencias Sociales. Instituto de Estudios para el Desarrollo Social; Trabajo y Sociedad; 32; 1-2019; 609-624.

Instituto Nacional de Estadística y Censos. (2021). Trabajo e ingresos. Vol. 5, № 4. Mercado de trabajo. Tasas e indicadores socioeconómicos (EPH). Informes técnicos / Vol. 5, N 118.Ciudad Autónoma de Buenos Aires: INDEC.

Garino, D. (2020). Entre la escuela y el trabajo: trayectorias educativo-laborales de egresados/as de escuelas secundarias. Neuquén: Topos

Jacinto, C. (Coord.). (2018). El secundario vale. Saberes, certificados y títulos técnicos en la inserción laboral de jóvenes. Buenos Aires: Miño y Dávila.

Maynard, A. (2019). Barreras discriminatorias en la demanda de empleo joven de la región de AMBA: un recorrido analitico entre los años 2009 y 2015. [Tesis de Maestría]. Buenos Aires: FLACSO.

Miranda, A. (2008). "Los jóvenes, la educación secundaria y el empleo a principios del siglo XXI". Revista de trabajo, 4(6), 185-198.

Miranda, A., Corica, A., Aranciba, M., y Merbilhaá, J. (2014). “Educación más trabajo $=$ menor desigualdad. La inserción educativa y laboral de los egresados 2011". Tiempos contingentes: inserción laboral de los jóvenes en la Argentina posneoliberal. Buenos Aires: Miño y Dávila.

Miranda A. (2015): Aportes para una lectura crítica del vínculo entre la juventud, la educación y el mundo del trabajo. En Miranda A. (edit): Sociología de la educación $y$ transición al mundo del trabajo: juventud, justicia y protección social en la Argentina contemporánea. Buenos Aires: Editorial Teseo.

Neffa, J. C., Panigo, D. T., Pérez, P. E., y Giner, V. (2000). Actividad, empleo y desempleo: conceptos y definiciones. Buenos Aires: Ceil-Piette Conicet.

Organización Internacional del Trabajo. (2020). Tendencias mundiales sobre empleo Juvenil 2020. Ginebra: OIT.

Rapoport, M. (2010). Historia económica, politica y social de la Argentina (1880-2003). Buenos Aires: Emecé. 
Scarano, A., Ascencio, D., Sacco, E., Gabilondo, F., Strada, J. y Ruiz Elvira, M. (2019). Mujeres argentinas. Entre la organización por la conquista de derechos y la profundización de las inequidades económicas. [Informe]. Centro de Economía Politica Argentina. Recuperado el 8 de julio de 2021, de https://gallery.mailchimp.com/e9c6f62a4dc825f6a9dab4e88/files/a1626eedbf86-406c-850e-57a7f6eabdc9/Mujeres_argentinas_8M_2019.pdf

Sennett, R. (2005). La corrosión del carácter. Las consecuencias personales del trabajo en el nuevo capitalismo. Barcelona: Anagrama.

Sosa, M., Smith, I. y Romano, D. (2021). Desempleo juvenil y políticas sociales. Los desafíos de la política pública para un problema estructural agravado por la pandemia. Recuperado el 16 de junio de 2021, de http://estudiosmetropolitanos.com.ar/wpcontent/uploads/2021/03/Desempleo-juvenil-y-pol\%C3\%ADticas-sociales_los-desaf\%C3\%ADos-de-la-pol\%C3\%ADtica-p\%C3\%BAblica-para-unproblema-estructural-agravado-por-la-pandemia.pdf

Tenti Fanfani, E. (1995). Títulos escolares y puestos de trabajo: elementos de teoría y análisis comparado. Revista Interamericana de Educación de Adultos 3 ( $N^{\circ}$ 2) Recuperado por última vez el 18 de noviembre de 2021, de http://planinst.unsl.edu.ar/pags-pdi/plan/1docs-info/docs-elec/tentimercados-de-trabajo.pdf

Terigi, F. (2007). Los desafíos que plantean las trayectorias escolares. Fundación Santillana. III Foro Latinoamericano de Educación Jóvenes y docentes. Recuperado el 13 de noviembre de 2021, de http://www.ieo.edu.ar/promedu/trayescolar/desafios.pdf.

Tiramonti, G. (2005). La reconfiguración de la educación en los últimos sesenta años. Revista Ñ. Buenos Aires: Club de Cultura Socialista José Aricó.

----. (2007). Subjetividades, pertenencias e intereses en el juego de la elección escolar. En Narodowski, M. y Gómez Schettini, M. (Comps.). Escuelas y familias. Problemas de la diversidad cultural y la justicia social. Buenos Aires: Prometeo.

Tuñón, I. y Halperin, V. (2010). Desigualdad social y percepción de la calidad en la oferta educativa en la Argentina urbana. Revista Electrónica de Investigación Educativa, 12(2). Recuperado el 14 de noviembre de 2021, de http://www.scielo.org.mx/scielo.php?script=sci_arttext\&pid=S1607$40412010000200005 \& \operatorname{lng}=\mathrm{es} \&$ tlng $=\mathrm{es}$

Weller, J. (2006). "Inserción laboral de jóvenes: expectativas, demanda laboral y trayectorias”. Boletín RedEtis, No 5, 1-6. Santiago de Chile: CEPAL.

----. (2007). "La inserción laboral de los jóvenes: características, tensiones y desafíos". Revista de la CEPAL No 92. Santiago de Chile: CEPAL. 